\title{
ПОВЫШЕНИЕ ТОЧНОСТИ ОДНОЗНАЧНОГО ИЗМЕРЕНИЯ СКОРОСТИ МЕТЕООБРАЗОВАНИЙ В ДОПЛЕРОВСКИХ МЕТЕОРАДИОЛОКАТОРАХ С ВОБУЛЯЦИЕЙ ИНТЕРВАЛОВ ЗОНДИРОВАНИЯ
}

\author{
ЛЕХОВИЦКИЙ Д. И. ${ }^{1}$, АТАМАНСКИЙ Д. В. ${ }^{2}$, РАЧКОВ Д. С. ${ }^{1}$, СЕМЕНЯКА А. В. ${ }^{1}$ \\ ${ }^{1}$ Харьковский нацииональный университет радиоэлектроники, \\ Украина, Харьков, 61166, пр-т Ленина, 14 \\ ${ }^{2}$ Харьковский университет Воздушных Сил имени Ивана Кожедуба, \\ Украина, Харьков, 61023, ул. Сумская 77/79
}

\begin{abstract}
Аннотация. Исследованы процедуры однозначного оценивания средней радиальной скорости метеообразований (МО) в импульсных доплеровских метеорадиолокаторах с постоянными и переменными (вобулированными) интервалами зондирования. Продемонстрировано, что выполнение требований к диапазону однозначного измерения скорости за счет вобуляции может сопровождаться нежелательным ростом ошибок ее оценивания. Определены причины этого недостатка в известных алгоритмах однозначного измерения скорости [1-4]. Показана возможность его ослабления за счет использования модифицированной оценки радиальной скорости. Исследовано влияние различных факторов (закон вобуляции, ширина спектра и порядок процесса авторегрессии, аппроксимирующего отражения от МО, объем обучающей выборки, вариант формирования финальной оценки скорости) на точность модифицированной оценки. Обоснованы рациональные параметры модифицированной процедуры и схема ее практической реализации
\end{abstract}

Ключевые слова: импульсный доплеровский метеорадиолокатор; однозначное оценивание средней радиальной скорости; вобуляция интервалов зондирования; процесс авторегрессии

\section{ВВЕДЕНИЕ И ПОСТАНОВКА ЗАДАЧИ}

Одна из основных задач импульсных доплеровских метеорадиолокаторов (ДМРЛ) распознавать опасные метеорологические явления, порождаемые ветром. Качество ее решения зависит, в частности, от точности определения скорости метеообразований (МO) в заданных элементах пространства, которая может измеряться различными способами [1-6]. В большинстве ДМРЛ применяется автоковариационный («пульспарный» $[1-4,7])$ метод измерения, в котором в роли радиальной составляющей $V_{r}$ этой скорости используется оценка $\hat{V}_{r}$ величины

$$
V_{r}=c \arg (r)
$$

$$
c=\lambda /(4 \pi T),
$$

где

$$
\arg (r)=\varphi_{T}=\operatorname{arctg}\left(r^{\prime \prime} / r^{\prime}\right)
$$

- аргумент (фаза) комплексного коэффициента корреляции (КК)

$$
\begin{gathered}
r=r^{\prime}+\mathrm{j} r^{\prime \prime}=R \exp \left(\mathrm{j} \varphi_{T}\right), \mathrm{j}=\sqrt{-1}, \\
R=\sqrt{\left(r^{\prime}\right)^{2}+\left(r^{\prime \prime}\right)^{2}}
\end{gathered}
$$

отсчетов отражений от МО, разделенных временным интервалом $T$.

Формула (1a) является следствием связи фазы КК 